\title{
Design and Testing of a Smartphone Application for Real-Time Self-Tracking Diabetes Self-Management Behaviors
}

\author{
Danielle Groat ${ }^{1,2}$ Hiral Soni ${ }^{2}$ Maria Adela Grando ${ }^{2}$ \\ Curtiss B. Cook ${ }^{2,3}$ \\ ${ }^{1}$ Department of Biomedical Informatics, University of Utah, Salt Lake \\ City, Utah, United States \\ 2 Department of Biomedical Informatics, Arizona State University, \\ Scottsdale, Arizona, United States \\ ${ }^{3}$ Division of Endocrinology, Mayo Clinic Arizona, Scottsdale, Arizona, \\ United States \\ Appl Clin Inform 2018;9:440-449.
}

Address for correspondence Maria Adela Grando, PhD, Department of Biomedical Informatics, Arizona State University, Scottsdale, Arizona, United States (e-mail: agrando@asu.edu).

\begin{abstract}
Keywords

- patient self-care

- smartphone

- usability testing

- survey

- diabetes

Background Type 1 diabetes (T1D) care requires multiple daily self-management behaviors (SMBs). Preliminary studies on SMBs rely mainly on self-reported survey and interview data. There is little information on adult T1D SMBs, along with corresponding compensation techniques (CTs), gathered in real-time.

Objective The article aims to use a patient-centered approach to design iDECIDE, a smartphone application that gathers daily diabetes SMBs and CTs related to meal and alcohol intake and exercise in real-time, and contrast patients' actual behaviors against those self-reported with the app.

Methods Two usability studies were used to improve iDECIDE's functionality. These were followed by a 30-day pilot test of the redesigned app. A survey designed to capture diabetes SMBs and CTs was administered prior to the 30-day pilot test. Survey results were compared against iDECIDE logs.

Results Usability studies revealed that participants desired advanced features for selftracking meals and alcohol intake. Thirteen participants recorded over 1,200 CTs for carbohydrates during the 30-day study. Participants also recorded 76 alcohol and 166 exercise CTs. Comparisons of survey responses and iDECIDE logs showed mean\% (standard deviation) concordance of $77 \%$ (25) for SMBs related to meals, where concordance of $100 \%$ indicates a perfect match. There was low concordance of $35 \%$ (35) and 46\% (41) for alcohol and exercise events, respectively.

Conclusion The high variability found in SMBs and CTs highlights the need for realtime diabetes self-tracking mechanisms to better understand SMBs and CTs. Future work will use the developed app to collect SMBs and CTs and identify patient-specific diabetes adherence barriers that could be addressed with individualized education interventions.
\end{abstract}

received

February 7, 2018

accepted after revision

April 24, 2018
DOI https://doi.org/ $10.1055 / \mathrm{s}-0038-1660438$. ISSN 1869-0327. 


\section{Background and Significance}

Type 1 diabetes (T1D) is a complex disease that affects 1.2 million people in the United States and has an estimated worldwide prevalence of 5 to $10 \%$ of all cases of diabetes. ${ }^{1,2}$ T1D is a chronic condition in which the pancreas does not produce insulin. The lack of insulin results in elevated blood glucose (BG). Effective treatment requires insulin injections before meals and throughout the day both to maintain BG as well as to offset above-target glucose levels. The goal of glycemic management is to maintain BG levels in a desired target range; avoiding high blood sugars (hyperglycemia) and low blood sugars (hypoglycemia). Poor glycemic control can lead to devastating complications including organ damage and increased mortality from heart disease. ${ }^{3,4}$ Patients who consistently engage in self-management behaviors (SMBs) and are adherent to standard treatment protocols are more likely to have better glycemic control. ${ }^{5}$ Examples of SMBs include monitoring BG, recording carbohydrate intake, administering insulin boluses (i.e., one large dose, generally between 1 and 15 units) to compensate for carbohydrate intake, and exercising. ${ }^{6}$ Tools such as insulin bolus calculators embedded into glucose meters and insulin pumps are designed to aid patients in making accurate insulin dosing decisions. However, even when these tools are available it is still difficult for patients to meet glycemic goals and little is understood about how patients include bolus calculators into their self-care beyond measuring frequency of use. ${ }^{7}$

Investigators have primarily studied self-reported data from surveys and interviews to typify SMB. ${ }^{5,8,9}$ It would be invaluable to understanding therapeutic and behavioral adherence if we could discern what the patient was thinking at a decision point, e.g. at the beginning of a meal or exercise routine, and the compensation techniques (CTs) employed at that moment to self-manage BG. While administering insulin is a $\mathrm{SMB}$, in this study the behaviors that the patient engages in at the point of consuming a meal or alcohol, or engaging in exercise, are considered CTs. Patients can employ one or more CTs at the time of an event which includes: doing nothing, consuming a snack, removing the pump, adjusting the basal rate (e.g., small dose of insulin released over time such as 1 unit per hour), administering an insulin bolus, or changing the bolus waveform (e.g. normal to square).

There is an abundance of smartphone applications (apps) that track exercise and nutrition for the general population, as well as apps that target patients with diabetes to facilitate the tracking of BG and medications. ${ }^{10}$ Insulin pumps automatically gather glucose readings and insulin-related data, but provide limited functionalities (e.g., patients only enter carbohydrates when they desire an insulin bolus suggestion from the bolus calculator) or limited tracking capabilities for other activities that impact BG, such as exercise or alcohol. Previously, the authors contrasted data collected from semistructured interviews against data collected by insulin pumps, glucose meters, continuous glucose monitors, and paper logs to understand the SMBs and CTs of adults with T1D with respect to alcohol, exercise, and meals. ${ }^{11,12}$ A limitation of that study was that participants did not always have the paper logs readily available to record SMBs related to alcohol, food, and exercise.
Replacing the paper logs for a smartphone app could increase the convenience for participants as they log behaviors which may improve the accuracy of the logs. To our knowledge, there are no apps for patients with T1D that allow them to track SMBs and CTs related to daily activities (e.g. eating a snack or disconnecting from the pump to compensate for exercise). ${ }^{10,13,14}$ Thus, we have limited insights into the cognitive processes associated with the management of their diabetes.

\section{Objectives}

The objectives of this study were (1) use a patient-centered approach to design iDECIDE, a smartphone app that gathers real-time diabetes SMBs and CTs related to meals, alcohol intake, and exercise, and (2) contrast patient's self-reported behaviors on a survey against self-tracked SMBs and CTs captured with iDECIDE.

\section{Methods}

Institutional Review Board approvals (\#1878, \#3516 and \#3689) were granted for the three phases of the usability testing and deployment of iDECIDE.

\section{Design Phase}

A literature search was conducted to gather information on the devices and software commercially available in the United States for T1D management. We engaged in discussions with endocrinologists and members of a diabetes care team regarding self-management challenges faced by patients with T1D. We participated in a simulated training session with a certified diabetes nurse educator that included hands-on experience with insulin pumps, glucose meters, and other related devices and software. Based what was learned regarding the impact that exercise, alcohol, and food have on BG control and T1D SMBs, iDECIDE's interfaces and functionalities were developed to achieve the following goals: (1) set up the user profile, such as define target BG, (2) set up SMB goals, such as exercise every day for 20 minutes, (3) self-track food and alcohol intake plans, including serving size, type, and carbohydrate content, (4) selftrack exercise plans, describing duration and intensity, (5) selftrack techniques used to compensate for food and alcohol intake and exercise, such as bolus insulin before food intake, and (6) support researchers' need to remotely access app data synchronized to cloud storage.

\section{iDECIDE First Usability Study}

iDECIDE was first built by the research team as a native Android app with Android Studio, the official integrated development environment for Android apps, and installed on a smartphone (-Fig. 1). ${ }^{15}$ We conducted a preliminary usability study with five healthy adult participants who did not have T1D, which was considered an adequate sample size to discover most problems for novel software. ${ }^{16}$ They were given a brief introduction to diabetes and presented with a fictitious T1D patient profile. Participants were given instructions to think aloud as they interacted with the app on the provided smartphone. ${ }^{17}$ Participants were allowed to explore the app for approximately 

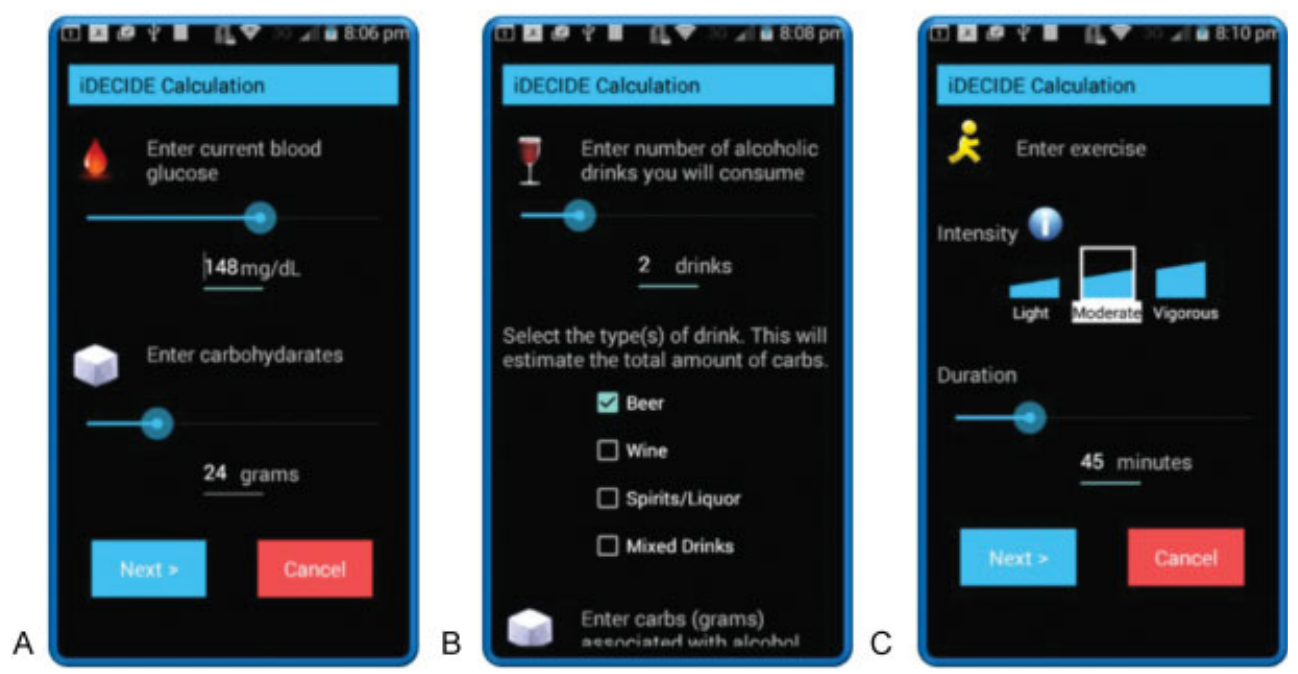

Fig. 1 Screenshots of the app from the first usability test. Participants could self-track (A) a meal's carbohydrates and current blood glucose, (B) the number and type of alcoholic beverages, and $(C)$ the duration and intensity of exercise. Design changes that resulted from the study included: removing the slider bars for data entry, changing check boxes into a pull-down menu, and providing additional features for tracking meals, alcohol, and exercise which required the design of additional icons.

5 minutes and then, one at a time, were given 7 tasks to complete. Two of the tasks were specific to self-tracking meals, alcohol, and exercise activities, while other tasks were related to setting up the user's profile or setting goals. The usability testing was recorded with Morae, a video capture and analysis tool, and the screencast of the smartphone was captured with Droid@Screen. ${ }^{18,19}$ Upon completion of the tasks, the participants were given a usability survey, which was modified from the System Usability Scale (SUS). ${ }^{20}$ The audio-video recordings were analyzed and annotated with Morae. Errors and comments were grouped into themes and the time to complete tasks was measured.

\section{iDECIDE Second Usability Study}

The feedback from the first usability study was used by the research team to improve the interfaces and functionality of iDECIDE. A prototype was built using Justinmind, a prototyping tool for Web and mobile apps, and tested by patients with $\operatorname{T1D}^{21}$ (- Fig. 2).

Although these six participants were adult patients with T1D, they were provided a fictitious T1D profile, similar to the first usability study. They were instructed to think aloud as they interacted with the app during the 5-minute exploration phase and while completing 7 tasks, one at a time. As

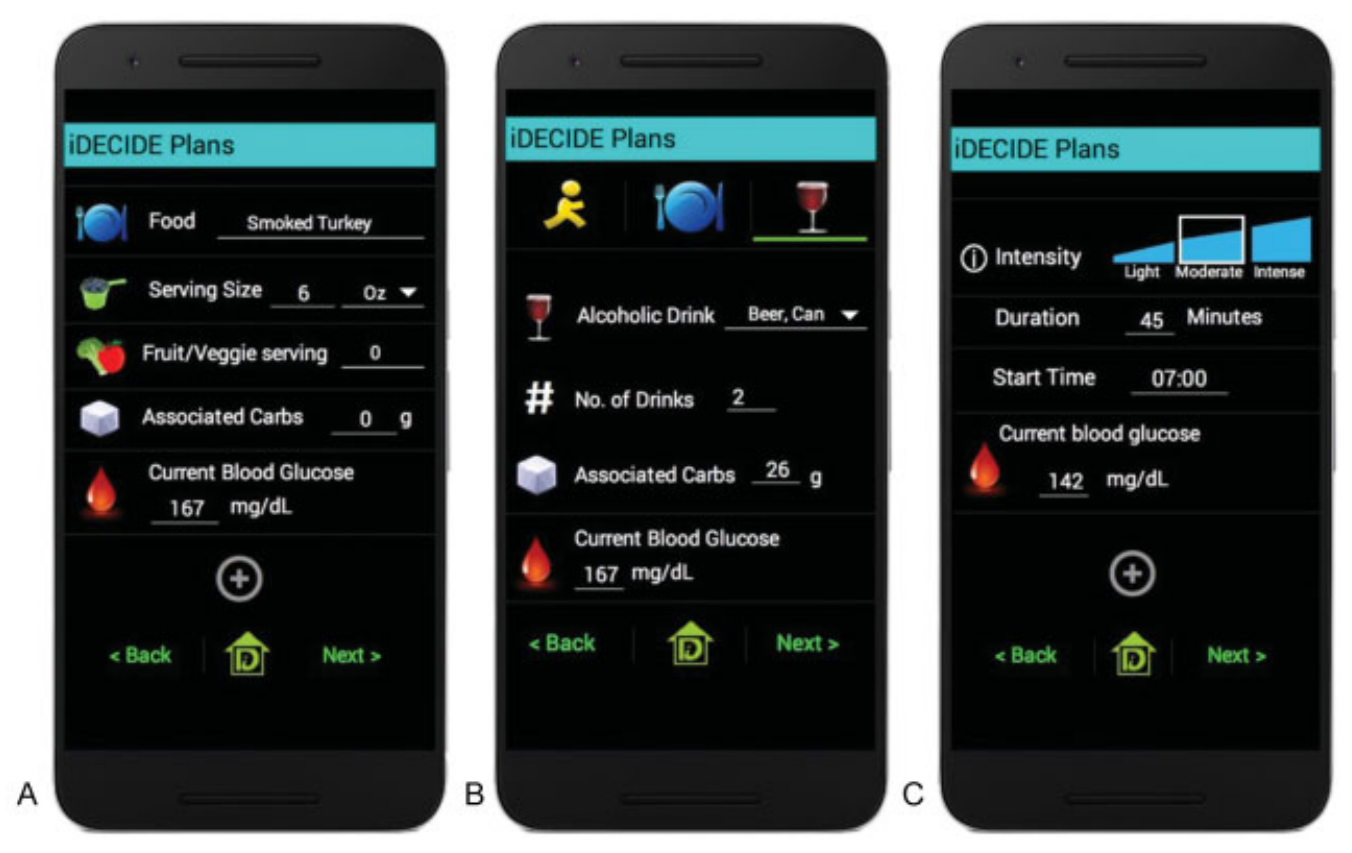

Fig. 2 Screenshots of the app from the second usability test. Participants could self-track (A) a meal item's serving size, number of fruit and vegetable servings, associated carbohydrates, and current blood glucose; (B) the number and type of alcoholic beverage, associated carbohydrates, and current blood glucose; and (C) the duration, intensity, start time, and current blood glucose of exercise. Following this study, the entry of meals and alcohol was streamlined from two interfaces down to just one interface and users could access the United States Department of Agriculture nutrition database. Additionally, users could track the compensation techniques employed at the time of meals and exercise. 
before, two of the tasks were specific to self-tracking meals, alcohol, and exercise. Other tasks dealt with app functionalities related to the user's profile and setting goals. After completing the tasks, the participants were given the same usability survey. Morae was used to record the usability sessions and to analyze the recordings.

\section{Self-Management Survey Design}

The survey content was informed by results from semistructured interviews and analysis of corresponding diabetes device-recorded data collected from patients with T1D. ${ }^{11,12}$ The objective of the survey was to provide more insight regarding (1) insulin pump usage behaviors, (2) reported SMBs and CTs for meals, exercise, and alcohol, and (3) perceptions of the effects of exercise and alcohol on BG control. The survey consisted of a combination of 11 structured, 14 semistructured, and 8 free-text questions (see - Supplementary Material: iDECIDE Recruitment Survey, available in the online version). Questions that inquired about specific techniques participants employed when compensating for meals, alcohol consumption, and when engaging in exercise were used for the analysis described in the "Comparison of Survey Responses versus Self-Tracking iDECIDE Logs" section. The questions did not ask about the frequency of any behaviors, but rather inquired how they currently self-manage in certain situations. Many of the CTs were automatically gathered from structured survey questions, while additional CTs were identified by manual review of free-text portions of the survey. The selfmanagement survey was administered electronically in the clinic prior to the pilot testing of the iDECIDE app.

\section{iDECIDE Pilot Testing}

Fourteen adult patients with T1D on insulin pump therapy were recruited to participate in the study. There were exclusion criteria for portions of study reported in Groat et $\mathrm{al}^{22}$ that limited the recruitment to patients using an insulin pump from a specific manufacturer. After consenting, participants were administered the self-management survey in an electronic format at the clinic. The app, which was developed by the research team, was then installed on their personal smartphone. Participants were instructed how to use the app. For the following 30 days the participants were asked to use iDECIDE to track meals, exercise activity, and alcohol intake. For each reported event, participants recorded one or more CTs (-Fig. 3) which were later used for comparison against the survey (the "Comparison of Survey Responses versus Self-Tracking iDECIDE Logs" section). The logs were synchronized to a secure cloud-based server. At the conclusion of the study, 9 of the 14 responded to a Web link after completing the pilot test to take an exit survey on the usability of iDECIDE.

\section{Comparison of Survey Responses versus Self-Tracking iDECIDE Logs}

Responses related to CTs from the self-management survey and self-tracking logs from the pilot test of iDECIDE were quantified. We examined concordance between survey responses and self-tracking logs. The CTs reported by each participant from the survey were noted separately for meals, alcohol, and exercise. In the case of meals, the total number of meals reported by the participant was used to determine the percentage of meals for which there were one or more matches with the survey. A concordance of $100 \%$ indicates that every meal reported by the participant with iDECIDE had at least one CT that matched one of the CTs reported on the survey for that event type.
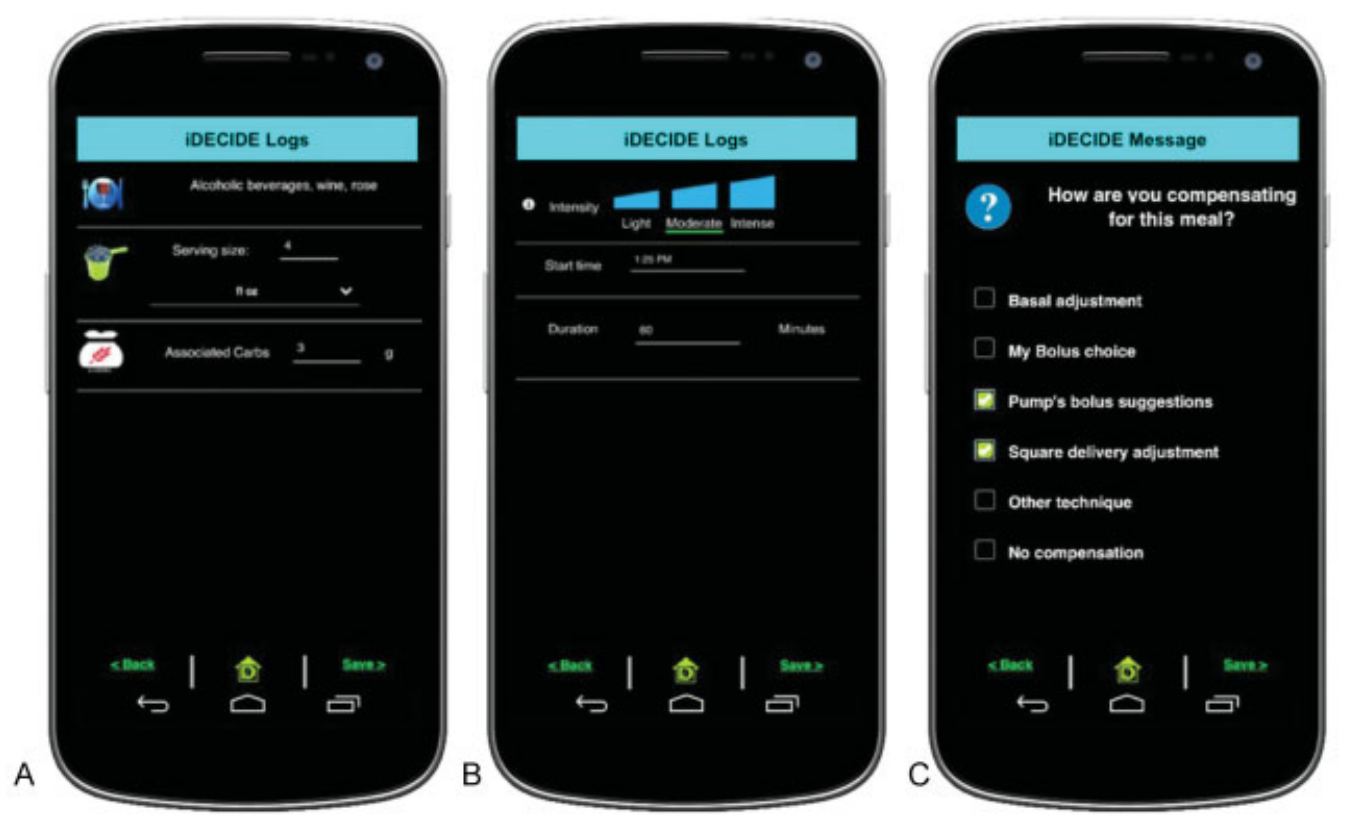

Fig. 3 Screenshots of the app from the 30-day pilot study. Participants could (A) self-track carbohydrate content of meals and alcoholic beverages, (B) self-track the start time, duration, and intensity of exercise, and (C) indicate the compensation techniques employed at the time of a meal, alcohol, or exercise activity. Following the pilot study, the app was designed for the Apple iOS platform and was also updated to provide advanced meal and alcohol tracking features. 


\section{Results}

\section{iDECIDE: First Usability Study}

Five graduate students were recruited and reported an average of 5.5 years of smartphone ownership. The average number of errors and time to complete the two tasks related to self-tracking meals, alcohol, and exercise are shown in - Table 1. Each task took an average of approximately 2 minutes to complete, with 2.0 errors occurring when tracking a meal and 3.4 errors occurring when tracking exercise. As the audio-video recordings were analyzed, usability issues emerged that were related to self-tracking, such as lack of flexibility for entering nutritional information for meals and difficulty viewing the numeric values beneath the slider bars ( - Table 1 ). The average subjective usability rating from the SUS questionnaire was 76.4, which can be interpreted that the app was good and acceptable, where a score of 100 indicates the best imaginable and acceptable. ${ }^{23}$

This preliminary study was instrumental in the iterative design of the app. Following this study and prior to conducting the second usability study, slider bars were removed and check boxes ( - Fig. 1B) were replaced with pull-down menus ( - Fig. 2B). More granularity was added to the tracking of meals, alcohol, and exercise which required the creation of additional icons, such as a measuring cup to represent serving size (-Fig. 2B). A food description became part of logging meals and alcohol and more than one food item could be logged at one time ( - Figs. 2A and B), for example, a single meal entry could consist of soup, breadsticks, and a beverage. Specifying a start time for exercise was added and more than one exercise segment could be tracked such that a single exercise event could consist of 10 minutes of light exercise at 7:00 a.m. followed by 20 minutes of moderate exercise at 7:10 a.m.

\section{iDECIDE Second Usability Study}

Six adults with T1D receiving care from an outpatient endocrinology department were recruited. The average years of smartphone ownership was 6.5. The average time to complete each task related to self-tracking of meals, alcohol, and exercise and the number of errors per task are presented in - Table 1. Usability issues related to the self-tracking tasks are also listed in - Table 1. The time to track a meal took longer than in the first usability study, and this can be attributed to the additional meal tracking features that were implemented. There were fewer errors for tracking exercise and alcohol in the second usability study, suggesting that the design changes were impactful. The main problem was the confusion participants had differentiating between short-term plans (e.g., drink one cocktail tonight) and goals (e.g., drink less than two units of alcohol every weekend). The average subjective usability rating from the SUS questionnaire was 79.9, which indicates the app was good and acceptable.

There were two problems identified in the second usability testing which required design changes that were implemented prior to the pilot test. First, to resolve issues related to differentiating plans from goals, the app's references to recording plans ( - Figs. 2A and B) were replaced with the notion of logs (-Figs. 3A and B). Second, the process for tracking meals and alcohol was streamlined into one interface $(-$ Fig. $3 \mathrm{~A})$ instead of two separate interfaces ( $\rightarrow$ Figs. $2 A$ and B). Due to the prototype environment, carbohydrate counting assistance was not implemented during the usability study but was included in the pilot-test of the app. Conversely, providing a current BG reading was part of the second usability study (-Figs. 2A-C) but not the pilot study (-Figs. 3A and B). The United States Department of Agriculture (USDA) nutrition database was accessible via text search within the app and populated the food description, serving size, and carbohydrates of meals and alcohol ( - Fig. 3A). Also implemented in the pilot test was the addition of self-tracking CTs for meals, alcohol, and exercise (-Fig. 3C).

\section{iDECIDE Pilot Test}

Fourteen adults with T1D on insulin pump therapy were recruited and completed the survey on SMBs. One

Table 1 Average duration in minutes, number of errors, and usability issues identified for each self-tracking task with the iDECIDE app

\begin{tabular}{|c|c|c|c|}
\hline Task & Duration (min) & \# of Errors & Usability issue \\
\hline \multicolumn{4}{|l|}{ First usability study } \\
\hline $\begin{array}{l}\text { Self-track meal with } \\
\text { alcoholic beverage }\end{array}$ & $\begin{array}{l}1.95 \\
(0.74)\end{array}$ & $\begin{array}{l}2.0 \\
(0.71)\end{array}$ & $\begin{array}{l}\text { Icons confusing, } \\
\text { functionality missing, } \\
\text { slider bar inconvenient }\end{array}$ \\
\hline Self-track exercise & $\begin{array}{l}2.24 \\
(0.76)\end{array}$ & $\begin{array}{l}3.4 \\
(1.52)\end{array}$ & $\begin{array}{l}\text { Functionality missing, } \\
\text { slider bar inconvenient }\end{array}$ \\
\hline \multicolumn{4}{|l|}{ Second usability study } \\
\hline $\begin{array}{l}\text { Self-track meal with } \\
\text { nonalcoholic beverage }\end{array}$ & $\begin{array}{l}2.56 \\
(0.53)\end{array}$ & $\begin{array}{l}1.3 \\
(0.5)\end{array}$ & $\begin{array}{l}\text { Goals versus plans } \\
\text { confusing }\end{array}$ \\
\hline Self-track exercise & $\begin{array}{l}1.35 \\
(0.74)\end{array}$ & $\begin{array}{l}0.3 \\
(0.5)\end{array}$ & $\begin{array}{l}\text { Goals versus plans } \\
\text { confusing }\end{array}$ \\
\hline
\end{tabular}

Note: All values are reported as mean and standard deviation (SD). 
participant withdrew from the study. Nine participants responded to the usability exit survey.

On the exit survey, participants were asked to rate several features of the iDECIDE app with a 1 to 5 Likert scale rating, with 5 being a positive rating. The average rating across all questions was 3.8. The responses on the survey were generally positive with most participants noting deficiencies with self-tracking meals. Users desired specific functionalities for logging meals, such as maintaining a list of favorites and more flexible searching capabilities for accessing the nutritional content of food from restaurants and grocers while the interface for tracking exercise was well received.
The app interfaces in - Fig. 4 depict how the desired functionalities were implemented. Users can use text searching to access: the USDA nutrition database, grocery data, and restaurant food data from the Nutritionix database, and in the case of alcohol, an internal database contains carbohydrate information for common drinks ( - Fig. 4A). ${ }^{24,25} \mathrm{~A}$ meal review page is provided which allows users to view all the components of the meal and to document the meal with a photo (-Fig. 4B). Unchanged from the pilot test, users indicate how, if at all, they are compensating for the meal, alcohol, or exercise (-Fig. 4C). Users can build a list of favorite foods to quickly populate the food description and
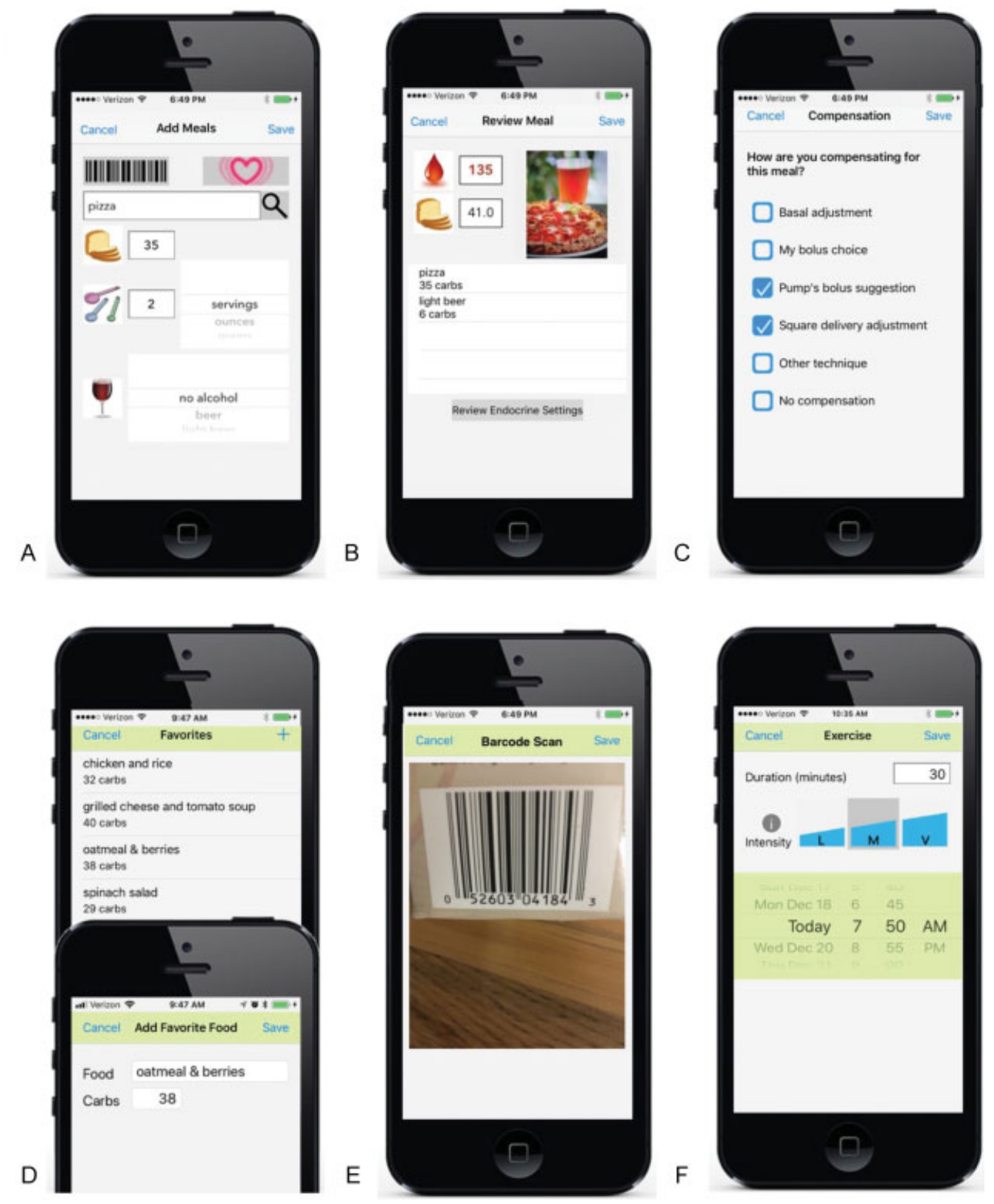

Fig. 4 Resulting app interfaces for the Apple platform based on user feedback includes the following functionalities for self-tracking meals: (A) carbohydrate counting assistance with textbox search function to access restaurant, grocery Nutritionix databases, and the United States Department of Agriculture food nutrition database, and an internal database of common alcoholic beverages; (B) blood glucose readings that sync from a HealthKit connected meter, meal component breakdown, and meal documentation with a photo; (C) compensation techniques employed for meals, alcohol, and exercise events; (D) favorite foods list defined by the user; and (E) barcode scanning to access grocery food data. The interface $(\mathbf{F})$ for tracking exercise duration, intensity and start time was relatively unchanged. 
carbohydrate content ( -Fig. 4D). Alternately, barcode scanning can be used to search grocery foods and their nutritional content ( - Fig. 4E). ${ }^{25}$ Relatively unchanged is the interface for self-tracking exercise (-Fig. 4F).

\section{Comparison of Survey Responses versus iDECIDE Self- Tracking Logs}

The survey helped to identify CTs for exercise and alcohol, along with reasons for employing those. Also, the survey identified factors that participants said affected BG control with regard to exercise and alcohol. Most importantly, the study confirmed the considerable inconsistency patients have about incorporating exercise and alcohol into decisions about SMBs. More details on the survey responses can be found at Groat et al. ${ }^{26}$ The comparison between the survey response and the iDECIDE self-tracking logs is detailed below.

\section{Carbohydrates}

Thirteen participants logged 1,710 meal events and 1,204 CTs with the iDECIDE app. The frequency of CTs self-tracked with the app for meals were: the pump's bolus suggestion (64.2\%), participant's bolus choice (14.7\%), no compensation (16.5\%), square wave bolus adjustment (3.9\%), basal adjustment $(0.4 \%)$, or other $(0.3 \%)$.

On average (standard deviation), there was $77 \%$ (25) concordance of the 1,204 meal events, where CTs the participants logged with the app matched to those that they had indicated on the survey. In the other $23 \%$ (25) of the meal events, participants' logged CTs differed from those reported in the survey (-Fig. 5A).

\section{Alcohol}

There were 10 participants that self-tracked alcohol intake with the app. Participants logged 86 alcohol events and 76 CTs. The CTs logged by the participants with the app when consuming alcohol were: following the pump's bolus suggestion (44.9\%), no compensation (34.6\%), participant's own bolus choice (15.4\%), square wave bolus adjustment (2.6\%), basal adjustment $(1.3 \%)$, or other $(1.3 \%)$.

Two of the CTs from the survey options did not match exactly with the selections available on the app. For the analysis of alcohol behaviors, we mapped the category "insulin bolus" from the survey to the app's choices of "pump's bolus suggestion" and "my bolus choice." "Eating a snack" from the survey was mapped to the app option "other technique." The inconsistency between the survey and the logs was higher for alcohol tracking than with meal tracking. There was very low and variable concordance of $35.4 \%$ (35) for the 86 alcohol events where participants' log entries matched their survey responses. Four participants reported on the survey that they did not compensate for alcohol in any fashion, but there were seven participants that self-tracked no compensation with the app ( - Fig. 5B).

\section{Exercise}

Thirteen participants logged a total of 181 exercise events along with 166 CTs. The CTs self-tracked with the app were:

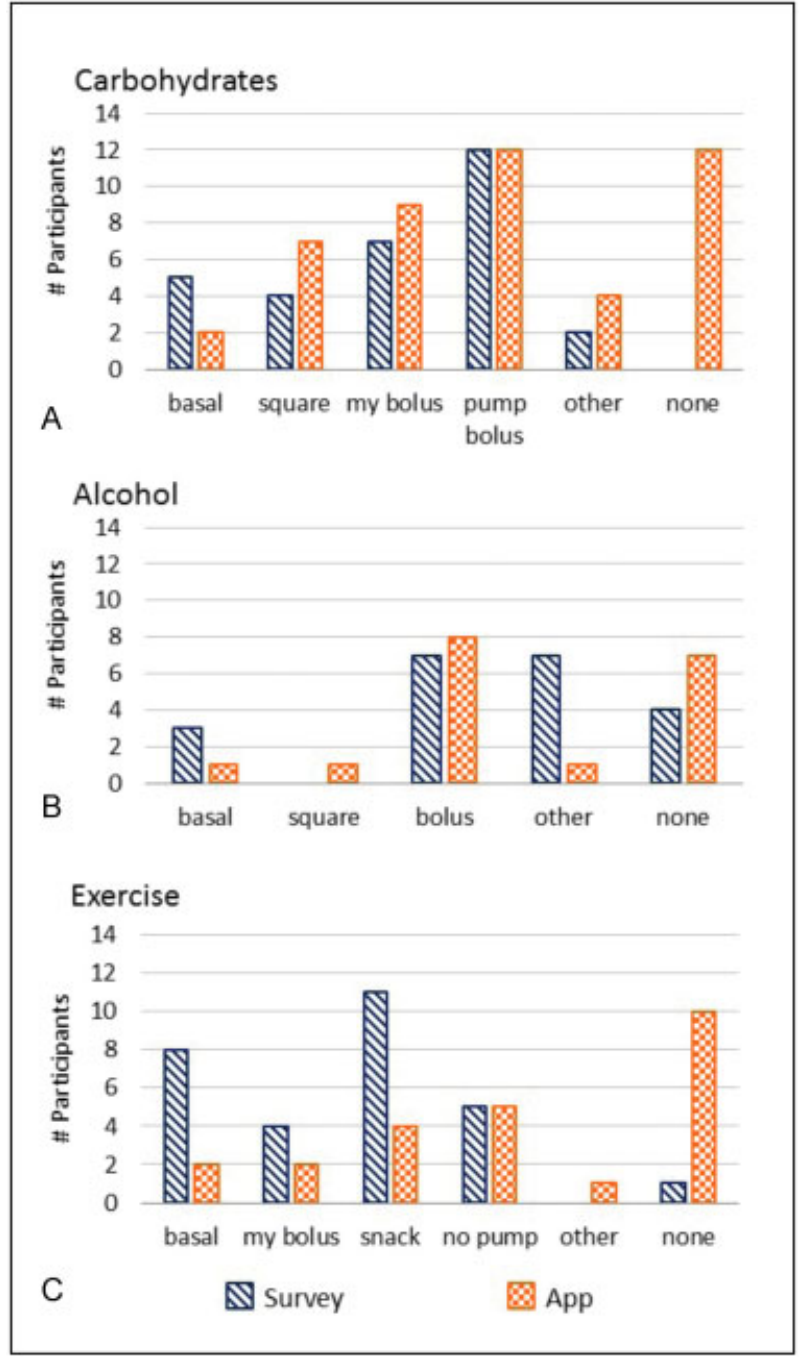

Fig. 5 Comparison of number of participants and their survey responses against self-tracking with the app for: (A) meals, (B) alcohol, and $(C)$ exercise. Compensation techniques were: basal adjustment (basal), square waveform bolus adjustment (square), participant's bolus choice (my bolus), pump's bolus suggestions (pump bolus), consume a snack (snack), disconnect from the pump (no pump), other technique (other), and no compensation (none).

no compensation (71.1\%), disconnecting from the pump (18.1\%), snack intake (4.8\%), basal adjustment (4.2\%), my bolus choice $(1.2 \%)$, or other $(0.6 \%)$.

The concordance for exercise had high variability and was found to be $46 \%$ (41) for the 181 exercise events. Eight participants reported adjusting the basal rate to account for exercise on the survey, but only 2 participants selftracked this technique in the app. Also, in the survey, 11 participants reported eating a snack while exercising, while only 4 participants self-tracked this technique with the app. -Fig. $5 \mathrm{C}$ depicts the discrepancies between survey responses and logs from the app regarding exercise.

\section{Discussion}

Most studies that aim to understand T1D SMBs rely on selfreported data gathered from surveys or interviews. ${ }^{5,8,26-31}$ 
While these studies are informative, they are subject to recall error (i.e., inaccurately remembering and reporting behaviors) and social desirability (i.e., overreport favorable behavior and underreport poor behavior). ${ }^{28,32}$ By using devices to facilitate real-time self-tracking, we can circumvent those limitations and gain additional insight into behavioral patterns. There are few freely available apps that support the tracking of $\mathrm{BG}$, insulin, meals, and exercise. ${ }^{13}$ Although there are many reviews that attempt to assess the current state of mobile applications used in randomized clinical trials of T1D, to the best of our knowledge there are no apps or diabetes devices that collect data on SMBs and CTs in real-time and at the level of granularity that has been done with iDECIDE for this study. ${ }^{14}$

The self-management survey and 30-day pilot test of iDECIDE is a continuation of previous work by the authors, ${ }^{11,12}$ where we replaced semistructured interviews with a more detailed survey and paper-based logs with a smartphone app for self-tracking meals, alcohol, and exercise, and for reporting CTs. When comparing behaviors from the survey and the app, we found a high level of concordance when compensating for meals and a high level of discordance and variability for alcohol and exercise. A limitation of the survey was asking participants about their current SMBs and not inquiring about their frequency. Also, the survey was administered before self-tracking with iDECIDE, and it is possible that concordance levels could have been higher if the survey was administered after pilot testing iDECIDE as participants may have reacted to the self-tracking by changing behaviors. Nonetheless, the results from this study and the aforementioned studies indicate that patients may misreport behaviors, particularly on surveys, but more importantly they indicate a need for developing better educational tools and programs to improve patients' ability to understand the effects of lifestyle choices, such as exercise and alcohol, which may lead to better glucose control.

Out of the 1,000+ diabetes apps available for download, it is unknown how many undergo usability testing, and it is unknown if patients or clinicians were involved during the design phases. ${ }^{33,34}$ The users that participated in the various studies described herein had substantial experience with smartphones and were highly educated and this may have influenced their desires for more features to be included with the app, which contradicts research that indicate fewer features improve the usability of diabetes apps. ${ }^{35}$ Further testing with users that have less smartphone experience or who do not use insulin pumps is needed to ensure that the advanced features of iDECIDE do not hinder usability for all patients with T1D. Based on two usability studies and user feedback from the 30-day pilot test, several new features were included for tracking meals and assisting with carbohydrate counting: carbohydrate content of common alcoholic beverages, barcode and text search access to food databases (e.g., USDA, restaurants, and grocery), user-generated favorite foods list, and photograph documentation of meals (-Fig. 4). iDECIDE also seamlessly shares data with Apple's HealthKit, including glucose readings, exercise, nutrition, and body weight. ${ }^{36}$ Furthermore, the data locally stored by iDECIDE is also synchronized with cloud services and can be remotely accessed through a password-protected Web-based interface.

Although this study did not assess the effectiveness of the app in improving outcomes nor did we address the need for better educational tools, this study and others have found that adherence to expected behaviors varies greatly and that undesirable behaviors frequently occur. $5,11,12,26,27$ Current behavioral interventions are based on the "one size fits all" approach which standardizes diabetes education. ${ }^{37}$ Group diabetes education has been compared against individualized instruction, but those studies did not use patient-reported data to influence individualized instruction. ${ }^{38}$ Initially, our goal in designing iDECIDE was to improve postprandial BG by accounting for meals, exercise, and alcohol. ${ }^{22}$ We realized that iDECIDE could easily be extended to gather real-time data that would help us better understand behaviors at the time decisions are made. With iDECIDE as a tool to measure CTs, we are now conducting a pilot study to identify unique self-care profiles based on SMBs and CTs logged in real-time. Individualized data-driven diabetes education will then be delivered which will be followed by self-tracking with iDECIDE for a second time to measure any changes in behaviors.

\section{Conclusion}

This study contrasted diabetes self-care behaviors of adults with T1D as self-reported on a survey against logs generated by self-tracking in real-time with the iDECIDE app. The study found inconsistencies between CTs reported by surveys and CTs documented with the app. Clinicians should use caution when interpreting patient's survey responses regarding exercise and alcohol behaviors when assessing adherence. The insights gathered from this study reveal the need for improved education and decision support tools to help patients on insulin therapy integrate alcohol and exercise behaviors as they self-manage BG. iDECIDE is currently being used in a pilot study to determine the effectiveness of datadriven educational interventions in improving SMBs.

\section{Clinical Relevant Statement}

A user-friendly, smartphone app that empowers T1D patients to track in real-time their lifestyle choices and diabetes selfmanagement behaviors (SMBs) and compensation techniques (CTs) related to food, alcohol, and exercise was designed and pilot tested.

Inconsistencies between SMBs and CTs reported on a survey and documented with the app we developed indicate that patients may misreport both types of behaviors. Clinicians should use caution when making diabetes care decisions that require interpreting SMBs and CTs related to alcohol and exercise reported by patients through surveys.

\section{Multiple Choice Question}

When contrasting self-reported versus real-time diabetes compensation techniques (CTs) as captured by a survey and a 
smartphone app, which behaviors were found to be highly (more than $70 \%$ ) consistent?
a. Exercise behaviors
b. Sleep behaviors
c. Meal behaviors
d. Alcohol behaviors

Correct Answer: The correct answer is option c. Comparisons of survey responses and iDECIDE logs showed high concordance of $77 \%$ for CTs related to meals, but low concordance of $35 \%$ and $46 \%$ for alcohol and exercise events, respectively. Sleep behaviors were not quantified.

\section{Protection of Human and Animal Subjects}

This study was reviewed by the Arizona State University and Mayo Clinic Institutional Review Boards.

Funding

This research was supported by the 2018 Mayo Clinic Robert D. and Patricia E. Kern Center for the Science of Health Care Delivery and the Arizona State University Research Acceleration Grant.

\section{Conflict of Interest}

None.

\section{References}

1 National Center for Chronic Disease Prevention and Health Promotion. National Diabetes Statistics Report, 2017. Estimate of diabetes and its burden on the United States; 2017. Available at: https:// www.cdc.gov/diabetes/pdfs/data/statistics/national-diabetes-statistics-report.pdf. Accessed September 9, 2017

2 World Health Organization. Global Report on Diabetes; 2016. Available at: http://apps.who.int/iris/bitstream/handle/10665/204871/ 9789241565257_eng.pdf;jsessionid=093AECB2B68112A20B0A35E226E8DECB? sequence $=1$. Accessed April 9, 2018

3 Nathan DM, Genuth S, Lachin J, et al; Diabetes Control and Complications Trial Research Group. The effect of intensive treatment of diabetes on the development and progression of long-term complications in insulin-dependent diabetes mellitus. N Engl J Med 1993;329(14):977-986

4 Laing SP, Swerdlow AJ, Slater SD, et al. Mortality from heart disease in a cohort of 23,000 patients with insulin-treated diabetes. Diabetologia 2003;46(06):760-765

5 Hendricks M, Monaghan M, Soutor S, Chen R, Holmes CS. A profile of self-care behaviors in emerging adults with type 1 diabetes. Diabetes Educ 2013;39(02):195-203

6 Weinger K, Butler HA, Welch GW, La Greca AM. Measuring diabetes self-care: a psychometric analysis of the Self-Care InventoryRevised with adults. Diabetes Care 2005;28(06):1346-1352

7 Klupa T, Benbenek-Klupa T, Malecki M, Szalecki M, Sieradzki J. Clinical usefulness of a bolus calculator in maintaining normoglycaemia in active professional patients with type 1 diabetes treated with continuous subcutaneous insulin infusion. J Int Med Res 2008;36(05):1112-1116

8 Olinder AL, Nyhlin KT, Smide B. Clarifying responsibility for selfmanagement of diabetes in adolescents using insulin pumps-a qualitative study. J Adv Nurs 2011;67(07):1547-1557

9 Ritholz MD, Beverly EA, Weinger K. Digging deeper: the role of qualitative research in behavioral diabetes. Curr Diab Rep 2011; 11(06):494-502

10 Hood M, Wilson R, Corsica J, Bradley L, Chirinos D, Vivo A. What do we know about mobile applications for diabetes self-man- agement? A review of reviews. J Behav Med 2016;39(06): 981-994

11 Groat D, Grando MA, Soni H, et al. Self-management behaviors in adults on insulin pump therapy. J Diabetes Sci Technol 2017;11 (02):233-239

12 Grando MA, Groat D, Soni H, et al. Characterization of exercise and alcohol self-management behaviors of type 1 diabetes patients on insulin pump therapy. J Diabetes Sci Technol 2017;11(02):240-246

13 Brzan PP, Rotman E, Pajnkihar M, Klanjsek P. Mobile applications for control and self management of diabetes: a systematic review. J Med Syst 2016;40(09):210

14 Bellei EA, Biduski D, Cechetti NP, De Marchi ACB. Diabetes Mellitus m-Health Applications: A Systematic Review of Features and Fundamentals. Telemed E-Health. Available at: https://www.libertpub. com/doi/abs/10.1089/tmj.2017.0230. Accessed April 10, 2018

15 Android. Android Studio. Available at: https://developer.android. com/studio/index.html. Accessed June 29, 2016

16 Lewis JR. Evaluation of procedures for adjusting problem-discovery rates estimated from small samples. Int J Hum Comput Interact 2001;13(04):445-479

17 Virzi RA, Sorce JF, Herbert LB. A Comparison of Three Usability Evaluation Methods: Heuristic, Think-Aloud, and Performance Testing. Los Angeles, CA: Sage Publications; 1993:309-313

18 TechSmith. Morae. Available at: http://www.techsmith.com/ morae.html. Accessed November 25, 2015

19 Ribomation AB. Droid@Screen. Available at: http://droid-atscreen.org/. Accessed December 16, 2015

20 mHIMSS. Selecting a Mobile App: Evaluating the Usability of Medical Applications; 2012. Available at: http://www.himss. org/selecting-mobile-app-evaluating-usability-medical-applications-0? ItemNumber=28900. Accessed September 26, 2017

21 Justinmind. Available at: http://www.justinmind.com. Accessed June 29, 2016

22 Groat D, Grando MA, Thompson B, et al. A methodology to compare insulin dosing recommendations in real-life settings. J Diabetes Sci Technol 2017;11(06):1174-1182

23 Bangor A, Kortum PT, Miller JT. An empirical evaluation of the system usability scale. Int J Hum Comput Interact 2008;24(06): 574-594

24 United States Department of Agriculture Research Service. USDA Food Composition Database. Available at: https://ndb.nal.usda. gov/ndb/. Accessed April 27, 2017

25 Nutritionix. Nutritionix - Largest verified nutrition database. Nutritionix; 2017. Available at: https://www.nutritionix.com/. Accessed June 5, 2017

26 Groat D, Soni H, Grando MA, Thompson B, Cook CB. Self-reported compensation techniques for carbohydrate, exercise, and alcohol behaviors in patients with type 1 diabetes on insulin pump therapy. J Diabetes Sci Technol 2018;12(02):412-414

27 O'Connell MA, Donath S, Cameron FJ. Poor adherence to integral daily tasks limits the efficacy of CSII in youth. Pediatr Diabetes 2011;12(06):556-559

28 Beverly EA, Ganda OP, Ritholz MD, et al. Look who's (not) talking: diabetic patients' willingness to discuss self-care with physicians. Diabetes Care 2012;35(07):1466-1472

29 Ravert RD. Alcohol management strategies of college students with diabetes. Patient Educ Couns 2009;77(01):97-102

30 Barnard KD, Dyson P, Sinclair JM, et al. Alcohol health literacy in young adults with type 1 diabetes and its impact on diabetes management. Diabet Med 2014;31(12):1625-1630

31 Pinsker JE, Kraus A, Gianferante D, et al. Techniques for exercise preparation and management in adults with type 1 diabetes. Can J Diabetes 2016;40(06):503-508

32 Guilfoyle SM, Crimmins NA, Hood KK. Blood glucose monitoring and glycemic control in adolescents with type 1 diabetes: meter downloads versus self-report. Pediatr Diabetes 2011;12(06):560-566

33 Brown W III, Yen P-Y, Rojas M, Schnall R. Assessment of the Health IT Usability Evaluation Model (Health-ITUEM) for evaluating 
mobile health (mHealth) technology. J Biomed Inform 2013;46 (06):1080-1087

34 Bastawrous A, Armstrong MJ. Mobile health use in low- and highincome countries: an overview of the peer-reviewed literature.J $R$ Soc Med 2013;106(04):130-142

35 Arnhold M, Quade M, Kirch W. Mobile applications for diabetics: a systematic review and expert-based usability evaluation considering the special requirements of diabetes patients age 50 years or older. J Med Internet Res 2014;16(04):e104
36 Apple, Inc. Apple Developer: HealthKit; 2017. Available at: https://developer.apple.com/healthkit/. Accessed April 26, 2017

37 American Diabetes Association. Standards of medical care in diabetes-2016 abridged for primary care providers. Clin Diabetes 2016;34(01):3-21

38 Dinneen SF, O'Hara MC, Byrne M, et al; Irish DAFNE Study Group. Group follow-up compared to individual clinic visits after structured education for type 1 diabetes: a cluster randomised controlled trial. Diabetes Res Clin Pract 2013;100(01):29-38 